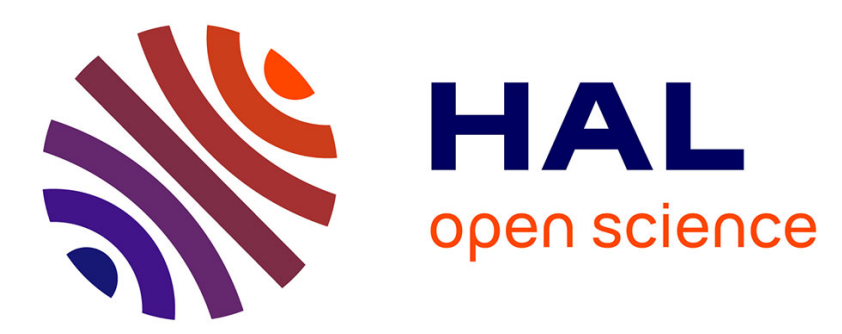

\title{
Kinematic modeling of an anthropomorphic hand using unit dual quaternion
}

\author{
Rohit Chandra, Juan Antonio Corrales Ramon, Youcef Mezouar
}

\section{To cite this version:}

Rohit Chandra, Juan Antonio Corrales Ramon, Youcef Mezouar. Kinematic modeling of an anthropomorphic hand using unit dual quaternion. 2019 IEEE/SICE International Symposium on System Integration (SII), Jan 2019, Paris, France. pp.433-437, 10.1109/SII.2019.8700387 • hal-02133782

\section{HAL Id: hal-02133782 \\ https://hal.uca.fr/hal-02133782}

Submitted on 19 May 2019

HAL is a multi-disciplinary open access archive for the deposit and dissemination of scientific research documents, whether they are published or not. The documents may come from teaching and research institutions in France or abroad, or from public or private research centers.
L'archive ouverte pluridisciplinaire HAL, est destinée au dépôt et à la diffusion de documents scientifiques de niveau recherche, publiés ou non, émanant des établissements d'enseignement et de recherche français ou étrangers, des laboratoires publics ou privés. 


\title{
Kinematic modeling of an anthropomorphic hand using unit dual quaternion*
}

\author{
Rohit Chandra, Juan Antonio Corrales-Ramon and Youcef Mezouar
}

\begin{abstract}
In this work we present the kinematic model of an anthropomorphic hand, consisting of fingers with coupled joints similar to humans, for forward and inverse kinematics computation. Additionaly, a strategy for relative pose control between the finger tips and thumb is also developed. The formulation uses geometrical analysis, and the kinematic model can easily be integrated with the kinematics of a robotic arm in order to benefit from the added redundancy. The forward and inverse kinematic formulations use unit dual-quaternion representation of screw based kinematics which ensures singularity free representation of orientation. The model hence obtained is implemented on an anthropomorphic hand for validation.
\end{abstract}

\section{INTRODUCTION}

The operation of robotic hands performing grasping tasks employs mainly two methods of operation: closing the fingers until the tactile sensors on the fingertips senses a contact; and using a kinematic model in conjunction with the tactile sensors. The availability of a kinematic model allows the user to pre-plan the grasping and in-hand manipulation tasks, if stable grasping locations that ensures force-closure grasps are known.

Underactuated hands provides a cheaper and lighter solution to grasping, in addition to self-adapting capability for different kinds of objects [1]. A four-bar linkage comprising middle and distal phalanx in a robotic finger can effectively imitate the coupling that exist between the proximal and distal interphalangical joints (i.e. PIP and DIP joints) of the human fingers. Additionaly, the higher load bearing capacity of four-bar linkages [2] makes them a natural choice for the purpose of effort transmission in fingers.

However, the mechanism employing these systems often presents additional complexity to the kinematic formulation, owing to the non-linear relation between the actuation and the motion of the finger joints. Some existing kinematic formulations for these kind of fingers uses a simplified linear coupling model for the relation between PIP and DIP joints at the cost of accuracy, or a complex actuation mechanism as a trade-off for the simplicity of the design [3].

In the current work we propose a geometrical method to obtain the relation between the finger joints actuation and the motion of finger consisting of such mechanisms using a coupling Jacobian. The formulation hence developed will allow the fingers to be used as an extension of the arm. Thus allowing for a combined (joints positions of the arm along with fingers joint positions) inverse-kinematics computation to ensure reachability of the fingertips to the

All authors are with Université Clermont Auvergne, SIGMA Clermont, Institut Pascal BP 10448, F-63000 Clermont-Ferrand, France.

Corresponding author is ol. chandra@gmail. com

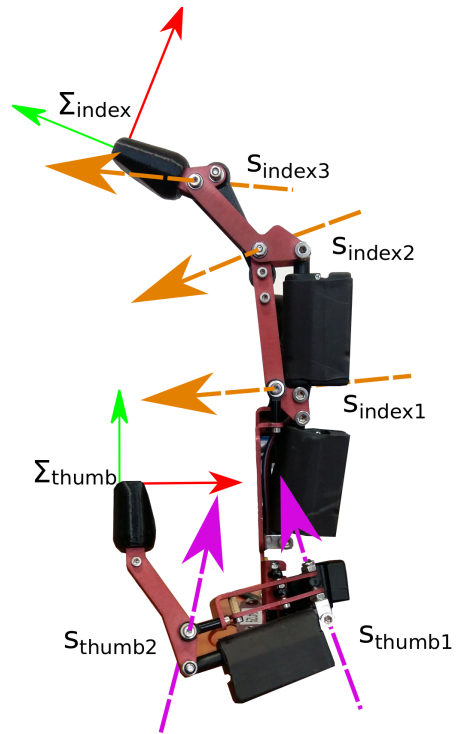

(a) Index finger and thumb of AR10 hand, with corresponding axes and frames.

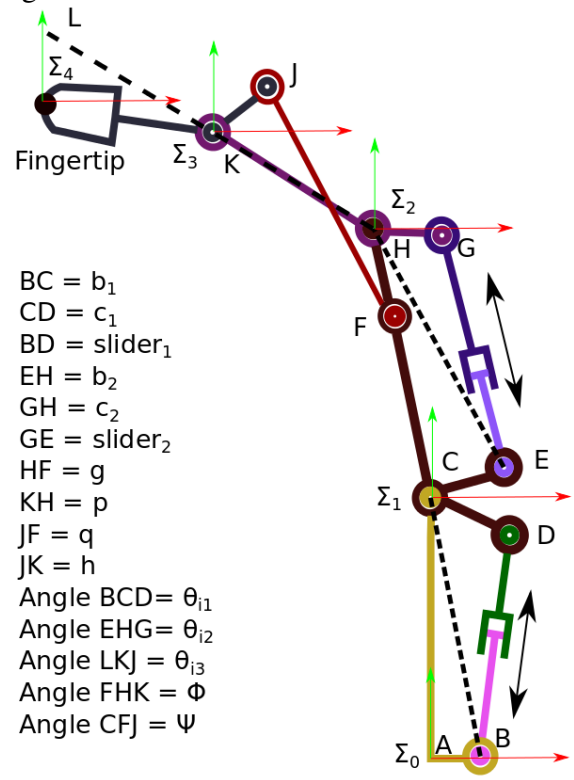

(b) Index finger mechanism: Frames defined on the base of the index finger $\left(\boldsymbol{\Sigma}_{\mathbf{0}}\right)$, on each rotational joints (i.e. $\left.\Sigma_{1}, \Sigma_{2}, \Sigma_{3}\right)$, and on the fingertip $\left(\Sigma_{4}\right)$.

Fig. 1: AR10 hand, and index finger mechanism.

grasping point, and pre-plan the grasping tasks in terms of the finger movement. In addition to that, a relative Jacobian has been derived between the index finger and the thumb 
(see Fig. 1a) using a screw-based method similar to [4], with the aim of using this model for grasping and in-hand manipulation.

The formulation hence developed has been validated on an anthropomorphic AR10 hand manufactured by Activ8 ROBOTS [5], consisting of fingers with lead screw actuators and four bar mechanisms (see Fig. 1a). Though AR10 hand provides a versatile yet relatively cost-effective solution for performing grasping and manipulation task, a kinematic model for the underactuated finger mechanism for forward and inverse kinematics currently does not exist, as far as the author knows.

In the following sections we describe the formulation of finger Jacobian using unit dual quaternion formulation. The use of screw based kinematics helps in avoiding the complexity of kinematic modelling associated with the traditional Denavit Hartenberg convention based method. Screw-based kinematics has the advantage of flexibility in the placement of frames on the links and selection of manipulator's reference configuration over Denavit-Hartenberg convention based kinematics [6]. Unit dual quaternions provide an efficient way to represent screw displacements, in addition to a singularity free representation of orientation [7].

Subsection II provides the detail of the formulation of kinematics and corresponding Jacobian for fingers with coupled joints, in addition to the formulation of relative Jacobian. The validation of the formulations obtained on AR10 hand is explained in sec. III. Afterwards, a summary of insights and future goals is presented in sec. IV.

\section{UNIT DUAL QUATERNION BASED KINEMATIC MODELING OF FINGER}

\section{A. Unit dual quaternion based kinematics}

The forward kinematics and Jacobian formulation using using unit dual quaternion representation presented in [7] has been briefed below for completeness. Notations used and respective meanings are defined as:

- Dual quaternions and dual vectors ${ }^{1}$ : Bold characters with hat (e.g. $\hat{\mathbf{x}}$ and $\hat{\mathbf{s}}$, respectively).

- Vectors: Bold characters (e.g. $l$ ).

- Row matrix: Underlined variables (e.g. $\underline{\boldsymbol{\theta}}, \underline{\hat{\boldsymbol{\theta}}}$ ).

Pose representation using unit dual quaternion is given as:

$$
\hat{\mathbf{x}}=\exp \left(\frac{\hat{\theta}}{2} \hat{\mathbf{s}}\right)=\cos \left(\frac{\hat{\theta}}{2}\right)+\hat{\mathbf{s}} \sin \left(\frac{\hat{\theta}}{2}\right)
$$

where, $\hat{\theta} \in \mathrm{D}$ is a dual angle and $\hat{\mathbf{s}} \in \mathrm{D}^{3 \times 1}$ is a directed line represented using a unit dual vector.

$$
\hat{\theta}=\theta+\varepsilon d, \quad \hat{\mathbf{s}}=\boldsymbol{l}+\varepsilon \boldsymbol{m}, \quad \varepsilon^{2}=0, \quad \varepsilon \neq 0,
$$

where, $\theta$ is a rotation around the screw axis, $d$ is translation along the same axis, $l$ is the unit direction vector of the screw axis, and $\boldsymbol{m}$ is the moment of this screw axis with respect

\footnotetext{
${ }^{1}$ Dual vectors are dual quaternions with pure quaternions as dual and non-dual part. A pure quaternion, $\boldsymbol{q}$ is defined as $\boldsymbol{q}=q_{1} \boldsymbol{i}+q_{2} \boldsymbol{j}+q_{3} \boldsymbol{k}$.
}

to the origin of the reference frame $(\boldsymbol{m}=\boldsymbol{p} \times \boldsymbol{l})$, and $\boldsymbol{p}$ is an arbitrary point lying on the screw axis.

Let $\hat{\mathbf{x}}_{e_{0}}$ be the initial pose of the end-effector with respect to the base frame, for the initial joint configuration $\underline{\hat{\boldsymbol{\theta}}}_{0}=\left[\begin{array}{llll}\hat{\theta}_{1_{0}} & \hat{\theta}_{2_{0}} & \ldots & \hat{\theta}_{n_{0}}\end{array}\right]^{T} \in \mathrm{D}^{n \times 1}$. The endeffector pose $\hat{\mathbf{x}}_{e}$, relative to the base frame, $\boldsymbol{\Sigma}_{\boldsymbol{b}}$, for joint configuration $\underline{\hat{\boldsymbol{\theta}}}=\left[\begin{array}{llll}\hat{\theta}_{1} & \hat{\theta}_{2} & \ldots & \hat{\theta}_{n}\end{array}\right]^{T} \in \mathrm{D}^{n \times 1}$ is given as:

$$
\begin{aligned}
\hat{\mathbf{x}}_{e} & =\boldsymbol{\delta}_{T} \hat{\mathbf{x}}_{e_{0}}, \\
\hat{\boldsymbol{\delta}}_{T} & =\hat{\boldsymbol{\delta}}_{1} \hat{\boldsymbol{\delta}}_{2} \cdots \hat{\boldsymbol{\delta}}_{n}, \\
\hat{\boldsymbol{\delta}}_{j} & =\exp \left(\frac{\hat{\theta}_{j}}{2} \hat{\mathbf{s}}_{j_{0}}\right) .
\end{aligned}
$$

where $\hat{\mathbf{s}}_{j_{0}}$ is the initial joint screw axis described using $\boldsymbol{l}_{j_{0}}$ and $\boldsymbol{m}_{j_{0}}, \hat{\theta}_{j}$ is a joint displacement from the home position $\hat{\theta}_{j_{0}}$, and,

$$
\begin{array}{lr}
\hat{\theta}_{j}=\Delta \theta_{j}, & \text { for revolute joints, } \\
\hat{\theta}_{j}=\varepsilon \Delta d_{j}, & \text { for prismatic joints. }
\end{array}
$$

The twist of the end-effector in the base frame, represented in the base frame, is given as:

$$
{ }^{b} \hat{\boldsymbol{\xi}}_{b e}=\boldsymbol{\omega}+\varepsilon \boldsymbol{v}_{o}
$$

where, $\boldsymbol{\omega}$ and $\boldsymbol{v}_{o}$, both pure quaternions, are the rotational velocity of the end-effector frame, and the translational velocity of a point which is attached to the end-effector frame and is instantaneously coincident with the origin of reference frame. The Jacobian using the current joint screw axis $\hat{\mathbf{s}}_{j}$ is given as:

$$
\hat{\mathbf{J}}=\left[\begin{array}{llll}
\hat{\mathbf{s}}_{1} & \hat{\mathbf{s}}_{2} & \cdots & \hat{\mathbf{s}}_{n}
\end{array}\right] .
$$

The dual vector of joint screw, $\hat{\mathbf{s}}_{j}$ for the new configuration, for the $i_{t h}$ joint, can be obtained from its initial value $\hat{\mathbf{s}}_{j_{0}}$, by transforming it using the total displacement caused by the previous $(j-1)$ joints ${ }^{2}$.

$$
\begin{gathered}
\hat{\mathbf{s}}_{j}={ }^{b} \hat{\boldsymbol{\delta}}_{T_{(j-1)}} \hat{\mathbf{s}}_{j_{0}}{ }^{b} \hat{\boldsymbol{\delta}}^{*} T_{(j-1)}, \\
{ }^{b} \hat{\boldsymbol{\delta}}_{T_{(j-1)}}=\hat{\boldsymbol{\delta}}_{1} \hat{\boldsymbol{\delta}}_{2} \cdots \hat{\boldsymbol{\delta}}_{(j-1)} .
\end{gathered}
$$

\section{B. Formulation of index finger Jacobian}

The kinematic mechanism corresponding to the index finger of AR10 hand (Fig. 1a) is given in Fig. 1b. The initial configuration of the joint angles, and screw axes are computed and stored for a desired starting configuration, with respect to the base frame of the index finger, i.e., $\boldsymbol{\Sigma}_{\boldsymbol{0}}$. The relation between the finger configuration and the slider positions is presented below.

The index finger consists of three joints where the last two joints are coupled. The corresponding joints are $\underline{\hat{\boldsymbol{\theta}}}_{\text {index }}=$ $\left[\begin{array}{lll}\hat{\theta}_{i 1} & \hat{\theta}_{i 2} & \hat{\theta}_{i 3}\end{array}\right]^{T}$. The proximal phalanx lead screw mechanism $\left(\right.$ slider $\left._{1}\right)$ rotates $\boldsymbol{\Sigma}_{1}$ around its $z$-axis, and thus

$$
{ }^{2 b} \hat{\boldsymbol{\delta}}^{*} T_{(j-1)} \text { is the classic dual quaternion conjugate of }{ }^{b} \hat{\boldsymbol{\delta}}_{T_{(j-1)}} .
$$


changing $\theta_{i 1}$. Using cosine rule for the $\triangle B C D, \theta_{i 1}$ is computed as:

$$
\theta_{i 1}=\cos ^{-1}\left(\frac{b_{1}^{2}+c_{1}^{2}-\operatorname{slider}_{1}^{2}}{2 b_{1} c_{1}}\right)
$$

$\theta_{i 2}$, and consequently the rotation of $\boldsymbol{\Sigma}_{2}$ caused by slider $_{2}$, is also computed using (8), using the corresponding parameters, i.e., $b_{2}, c_{2}$ and slider $_{2}$. The change in the slider $_{2}$ caused by the middle phalanx movement leads to the change in $\theta_{i 2}$ as well as $\theta_{i 3}$, owing to the four-bar mechanism of the distal phalanx and middle phalanx defined by the segments $H F, J F, J K$ and $K H$. As can be seen in the Fig. (1b), the change in $\theta_{i 2}$, also corresponds to change in $\phi . \theta_{i 3}$ corresponding to a given $\phi$ is obtained using the constraint of fixed length of the link $J K$, and the two loop equations to define the position of point $K$ from point $H$.

$$
\theta_{i 3}=\tan ^{-1}\left(\frac{q \sin (\psi)-p \sin (\phi)}{g+q \cos (\psi)-p \cos (\phi)}\right)-\phi
$$

where, $\psi$ is given as:

$$
\begin{aligned}
& \psi(\phi)=\tan ^{-1}\left(\frac{B}{A}\right)-\cos ^{-1}\left(\frac{C}{\sqrt{A^{2}+B^{2}}}\right) \\
& A(\phi)=2 p q \cos (\phi)-2 g q, \\
& B(\phi)=2 p q \sin (\phi) \\
& C(\phi)=g^{2}+p^{2}+q^{2}-h^{2}-2 p g \cos (\phi) .
\end{aligned}
$$

The relation between the derivative of $\phi$ or $\theta_{i 2}$, and $\theta_{i 3}$ is given as ([8]):

$$
\begin{aligned}
f\left(\theta_{i 2}, \psi, \theta_{i 3}\right) & =\frac{\dot{\theta_{i 3}}}{\dot{\phi}}=\frac{\dot{\theta_{i 3}}}{\theta_{i 2}} \\
& =\frac{a \sin (\psi-\phi)-h \sin \left(\phi+\theta_{i 3}-\psi\right)}{h \sin \left(\phi+\theta_{i 3}-\psi\right)} .
\end{aligned}
$$

Since change in $\theta_{i 2}$ corresponds to the change in $\phi$, the coupling Jacobian is given as $f\left(\theta_{i 2}, \psi, \theta_{i 3}\right)$, assuming the initial offset between $\theta_{i 2}$ and $\phi$ is known. Finally, the unit dual quaternion based Jacobian for the index finger is given as (see Fig. 1a):

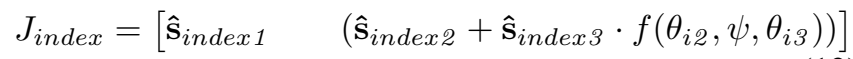

Please note that the relations (9) to (12) are only valid for the crossed configuration given in Fig. 1b ([9], [8]). It has been assumed that the configuration will be crossed throughout the operation, which is, in fact, true for the particular hand we have chosen for validation of the strategy.

The error unit dual quaternion $\hat{e}$ in the base frame, can obtained as the relative displacement between the desired and current pose, i.e. $\hat{\mathbf{x}}_{d}$ and $\hat{\mathbf{x}}_{c}$, respectively computed in the base frame.

$$
\hat{\mathbf{e}}=\hat{\mathbf{x}}_{c} \hat{\mathbf{x}}_{d}^{*}
$$

where, $\hat{\mathbf{x}}_{d}^{*}$ is the classical quaternion conjugate of $\hat{\mathbf{x}}_{d}$. The control law for kinematic control for screw-based method using unit dual quaternion representation for a given gain $\lambda$, is:

$$
\begin{aligned}
\hat{\boldsymbol{\xi}} & =-\lambda \ln (\hat{\mathbf{e}}) \\
& =-\lambda\left(\theta_{e} \boldsymbol{l}_{e}+\varepsilon\left(\theta_{e} \boldsymbol{m}_{e}+d_{e} \boldsymbol{l}_{e}\right)\right)=-\left(\boldsymbol{\omega}_{e}+\varepsilon \boldsymbol{v}_{o e}\right)
\end{aligned}
$$

where, $\left\{\theta_{e}, d_{e}, \boldsymbol{l}_{e}, \boldsymbol{m}_{e}\right\}$ are screw displacement parameter related to the error dual quaternion $\hat{e}$, and $\lambda$ is a positive scalar gain for the controller. The global exponential convergence of the above mentioned control law $(\hat{\xi})$ has been proved for $\left(-\pi \geq \theta_{e} \geq \pi\right)$ in [7].

\section{Formulation of relative Jacobian}

The thumb consists of two lead screw mechanisms with three bars, in series. The geometrical arrangement is similar to the first two mechanisms of the index finger, i.e., $\triangle B C D$ and $\triangle E H G$. However, the rotation axes of the thumb, i.e. $\hat{\mathbf{s}}_{\text {thumb1 }}$ and $\hat{\mathbf{s}}_{\text {thumb2 }}$, are not coplanar, as was the case with the index finger (refer Fig. 1b). The forward kinematics for the thumb is computed using (8).

The relative Jacobian maps the intermediate joint displacement to the relative screw displacements between the index fingertip and thumbtip frames. The index fingertip is taken as the reference frame for the relative pose control (Fig. 1a). The relative Jacobian defined in the index fingertip frame is given as:

$J_{r e l}=\left[\begin{array}{llll}\left(\hat{\mathbf{s}}_{i 2}+\hat{\mathbf{s}}_{i 3} \cdot f\left(\theta_{i 2}, \psi, \theta_{i 3}\right)\right) & \hat{\mathbf{s}}_{i 1} & \hat{\mathbf{s}}_{t 1} & \left.\hat{\mathbf{s}}_{t 2}\right)\end{array}\right]$

Please note that, in the above equation, the subscript index has been replace with $i$ for the joint screw axes of index finger, for the sake of compactness, whereas subscripts $t 1$ and $t 2$ refers to the joint screw axes of the thumb joints. It is important to point out that all the joint screw axes, which were computed at the base frame, $\boldsymbol{\Sigma}_{0}$, has to be transformed to the frame at the index fingertip $\left(\boldsymbol{\Sigma}_{\text {index }}\right)$, during the starting of the computation. The joint displacement array corresponding to the relative Jacobian defined in (15) is:

$$
\delta \underline{\hat{\boldsymbol{\theta}}}_{r e l}=\left[\begin{array}{llll}
-\delta \hat{\theta}_{i 2} & -\delta \hat{\theta}_{i 1} & \delta \hat{\theta}_{t 1} & \delta \hat{\theta}_{t 2}
\end{array}\right]^{T}
$$

Again, note that the joint displacements for the index finger joints are negative, in order to account for the reverse sense of displacement as seen from the index finger tip frame, compared to the displacement observed from the base frame $\Sigma_{0}$.

\section{EXPERIMENTAL VALIDATION AND RESULT}

The Jacobian formulated in (12) for the finger mechanism was used for the inverse kinematic control for the index finger of AR10 hand (see (14)). Using the formulations discussed in subsection II-B, the initial values of $\theta_{1}, \theta_{2}$, and $\theta_{3}$ were obtained. The subsequent angular displacements were computed using the current slider positions for forward kinematics. The slider commands for inverse kinematic control were computed by using the inversion of (8) and (9), using the initial and the computed values of $\theta_{1}, \theta_{2}$, and $\theta_{3}$. 


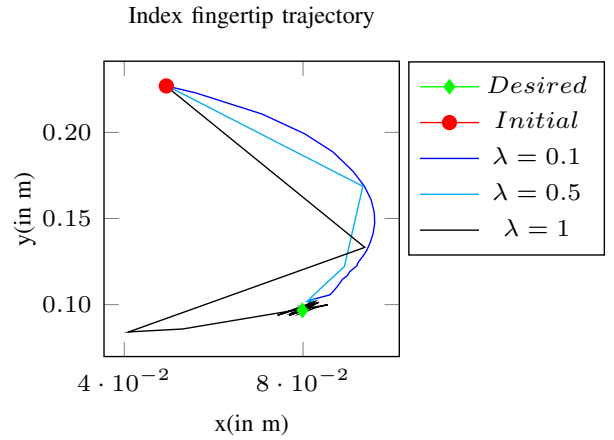

Fig. 2: Trajectory of the fingertip of the index finger.

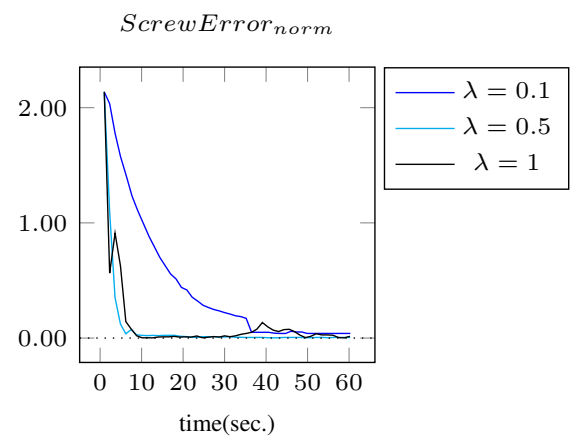

Fig. 3: Performance of the inverse kinematics controller.

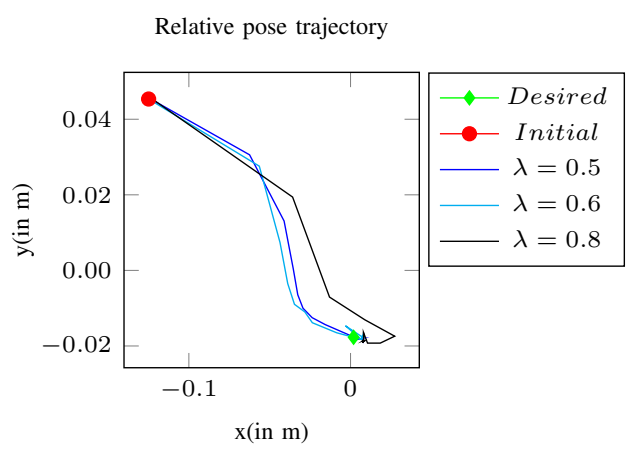

Fig. 4: Trajectory of the relative pose between index fingertip and thumbtip in the index fingertip frame.

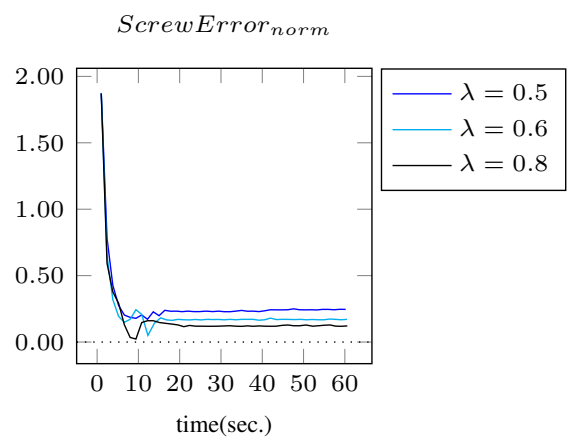

Fig. 5: Performance of the inverse kinematics controller for relative pose control.
TABLE I: Error of the index finger IK computation.

\begin{tabular}{|c|c|c|}
\hline Gain & $\mathrm{X}_{\text {error }}$ (in mm.) & Yerror $_{\text {(in } \mathrm{mm} \text {.) }}$ \\
\hline 0.1 & -1.37181 & -3.89763 \\
\hline 0.5 & -0.196327 & -0.373781 \\
\hline 1 & -0.598718 & -0.714857 \\
\hline
\end{tabular}

The parameters given in Fig. (1b) and similarly for the thumb, i.e. the dimensions of all the involved segments, were computed using manual measurements. In the following experiments we express the error of inverse kinematics with the assumption of perfect knowledge of those parameters. Since the fingers have very limited workspace, a pre-identified achievable configuration was chosen as desired position of the finger tip, which was given as unit dual-quaternion. As the finger can be considered as a planar mechanism, the $X Y$ plane trajectory taken by the finger tip have been depicted in Fig. 2.

The exponential convergence of the norm of the error screw parameters used in the controller (see (14)), computed as:

$$
\text { ScrewError }_{\text {norm }}=\sqrt{\boldsymbol{\omega}_{e}^{2}+\boldsymbol{v}_{o e}^{2}},
$$

is evident in Fig. 3. Among the three controller gains tested, the best performance was achieved for the gain $\lambda=0.5$. The convergence is slowest for $\lambda=0.1$, while some oscillation can be seen in the Finger tip trajectory plot in Fig. 3 for $\lambda=1$. The error at the end of the inverse kinematic control, i.e. at the steady state is given in Table I, which demonstrates that for the gains of $\lambda=0.5$ and 1 , the error is under one $\mathrm{mm}$.
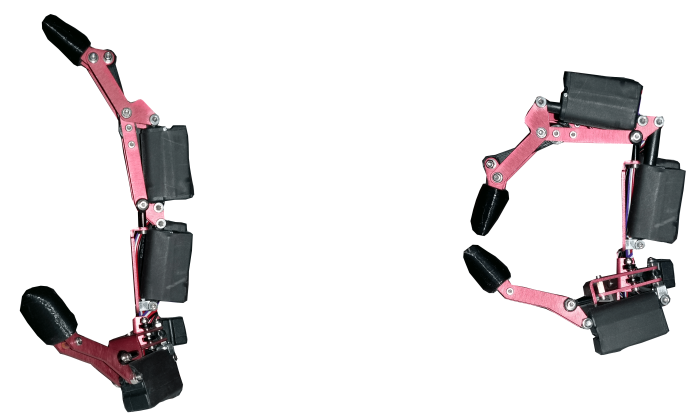

Fig. 6: Initial and Desired configuration of relative motion task.

The performance of the relative pose control between index and thumb tips are given in Fig. 4 and Fig. 5. The results for only those gains that provided satisfactory result have been provided. Similar to the index finger inverse kinematic control, the desired pose was obtained from a known achievable pose. As can be seen in Fig. 4, the gain of $\lambda=0.6$ was found to be most suited for relative pose control. However, an offset can be observed in the ScrewError norm 
in the Fig. 5, which is $5.5 \mathrm{~mm}$ and $0.2 \mathrm{~mm}$ in $x$ and $y$ axis respectively. This is attributed to the fact that the relative task space consists of 6 dof s, while the combined sytem of index and thumb can only afford $4 \operatorname{dof}$ s. While this was the same case with index finger, the planar construction of index finger helped dealing with issue. While in the case of thumb plus index finger, there are non-planar joints involved.

\section{CONCLUSiOn AND FUTURE WORK}

The finger Jacobian comprising of coupled joints used in the inverse kinematics controller demonstrates exponential convergence for the desired end-effector position. The relative pose control between index and thumb fingertip gave promising results, however demonstrated the need for further work, so that it can be used for real applications like in-hand manipulation.

For the future work, we have identified following areas of improvements:

- Calibration of fingers: As we noted in the experimental validation section, the parameters used in the modeling
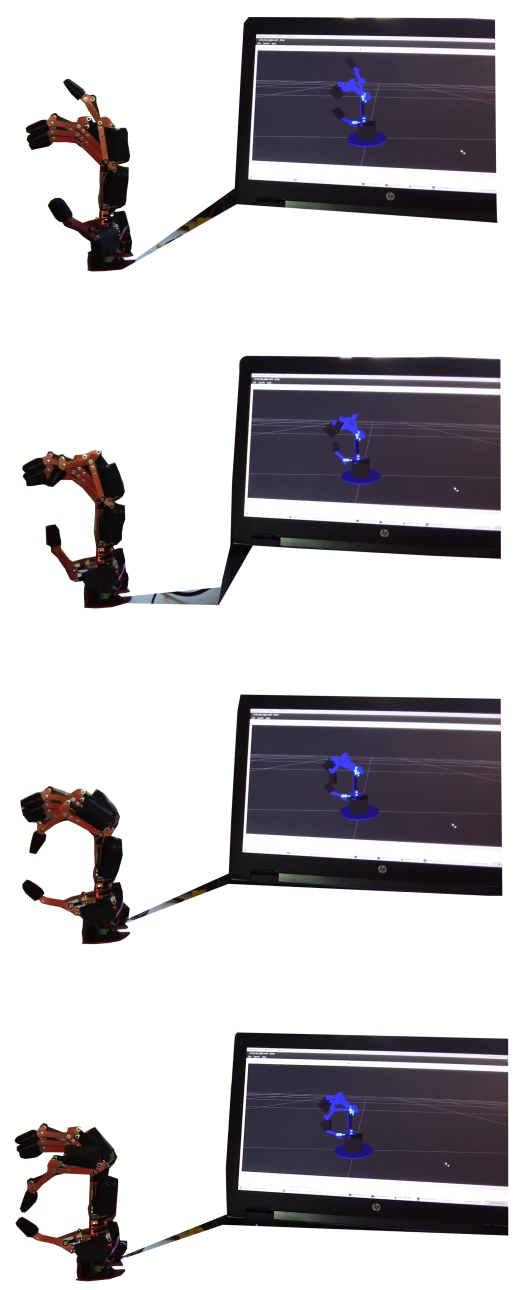

Fig. 7: Different stages of inverse kinematic control for relative pose. were manually measured, since the manufacturer have not provided an accurate model. A vision based calibration method can be employed to estimate the joint screw axes parameters and the fingertips poses [10].

- Task specific Jacobian: Since the fingers have limited $d o f$ s, special care has to taken about the control objectives. For example, distance Jacobians can be employed for relative control [11], since it is not really important to control the orientation of the frames, in the case of grasping. In addition to that, null-space control can be used along with reduced dimension tasks, to respect the joint limits of the fingers.

- Integration with a robotic arm: The kinematic model for the fingers developed in this work can easily be integrated with the kinematic model of a robotic arm, for example Baxter robot [12], to increase the applicability of the hand.

\section{ACKNOWLEDGMENT}

This work is funded by the European Union. The European Union gets involved in Auvergne-Rhône-Alpes through the European Regional Development Fund (FEDER).

\section{REFERENCES}

[1] G. A. Kragten, F. C. Van der Helm, and J. L. Herder, "A planar geometric design approach for a large grasp range in underactuated hands," Mechanism and Machine Theory, vol. 46, no. 8, pp. 1121$1136,2011$.

[2] A. K. Natesan, "Kinematic analysis and synthesis of four-bar mechanisms for straight line coupler curves," 1994.

[3] I. Godler, K. Hashiguchi, and T. Sonoda, "Robotic finger with coupled joints: A prototype and its inverse kinematics," in Advanced Motion Control, 2010 11th IEEE International Workshop on. IEEE, 2010, pp. 337-342.

[4] R. Guenther and D. Martins, "Screw-based relative jacobian for manipulators cooperating in a task," in ABCM Symposium Series in Mechatronics, vol. 3, 2008, pp. 276-285.

[5] "Ar10 humanoid hand. https://www.active8robots.com/robots/ar10robotic-hand/." [Online]. https://www.active8robots.com/robots/ar10-robotic-hand/

[6] J. Funda and R. P. Paul, "A computational analysis of screw transformations in robotics," IEEE Transactions on Robotics and Automation, vol. 6, no. 3, pp. 348-356, 1990.

[7] E. Özgür and Y. Mezouar, "Kinematic modeling and control of a robot arm using unit dual quaternions," Robotics and Autonomous Systems, vol. 77, pp. 66-73, 2016.

[8] J. M. McCarthy, Geometric design of linkages. Springer Science \& Business Media, 2006, vol. 11.

[9] G. Toussaint, "Simple proofs of a geometric property of four-bar linkages," The American mathematical monthly, vol. 110, no. 6, pp. 482-494, 2003.

[10] H. Wang, S. Shen, and X. Lu, "A screw axis identification method for serial robot calibration based on the poe model," Industrial Robot: An International Journal, vol. 39, no. 2, pp. 146-153, 2012.

[11] B. V. Adorno, "Two-arm manipulation: From manipulators to enhanced human-robot collaboration," Ph.D. dissertation, Université Montpellier II-Sciences et Techniques du Languedoc, 2011.

[12] R. Robotics, "Baxter research robot," Retrieved April, vol. 26, p. 2018, 2013. 